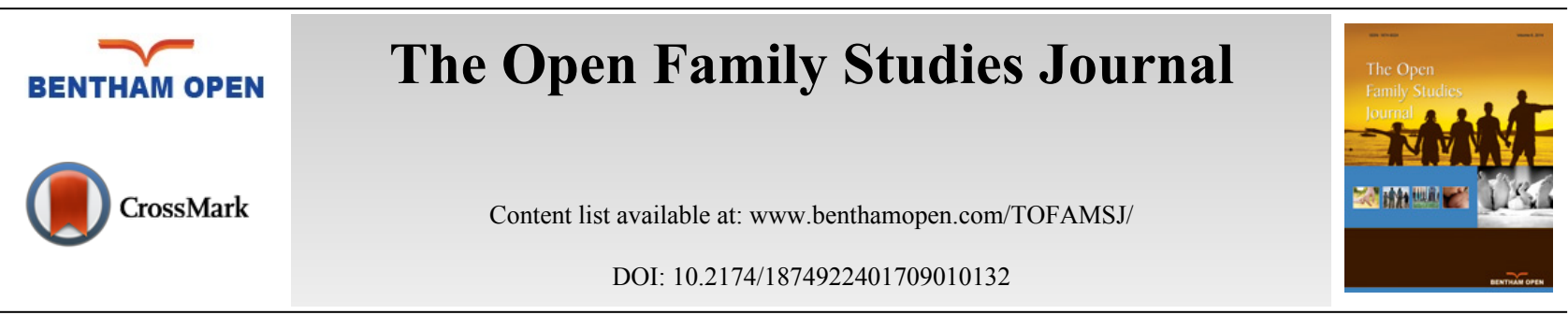

RESEARCH ARTICLE

\title{
Effectiveness of Therapeutic Attachment Camps for Improving Behavior in Children with Reactive Attachment Disorder
}

\author{
Anne M. Coleman ${ }^{1}$ and Allen Rand Coleman ${ }^{2, *}$ \\ ${ }^{1}$ Cabrini University, Radnor, PA 19087, USA \\ ${ }^{2}$ Cornerstone Therapy \& Wellness, Malvern, Pennsylvania, USA
}

Received: July 31, 2017

Revised: August 14, 2017

Accepted: September 14, 2017

\begin{abstract}
:
Background:

Early childhood attachment disruption manifests in disruptive, oppositional behavior and reduced ability for trusting intimate relationships. Chronic emotional disorder negatively affects the entire family system, making treatment difficult.
\end{abstract}

\section{Objective:}

The goal of the study was to assess a family therapeutic treatment program for children with Reactive Attachment Disorder (RAD) conducted through an intensive, one-week camp.

\section{Methods:}

Therapeutic camps included training for caregivers, support for siblings, behavioral interventions for children with RAD, and family therapy exercises. Camps were conducted across North America. Standardized behavioral health rating scales were used to evaluate outcomes.

\section{Results:}

Therapeutic Attachment Camp effectively reduced disruptive behaviors within a one week period and improved family mental health scores. Pretest to post-test scores on the Randolph Attachment Disorder Questionnaire showed significant change in a clinically positive direction. Behaviors specifically associated with attachment and conscience development improved, such as showing remorse or guilt, self-control, telling the truth and accepting parental direction. Child anxiety was observed to be less based on selfrating on the State-Trait Anxiety Inventory. Parent anxiety was significantly reduced based on the State-Trait Anxiety Inventory. Statistical effects were moderate to large.

\section{Conclusion:}

Results suggested that a treatment program addressing the needs of the entire family and combining attachment exercises with psychoeducation and structured parenting practice can be effective within a short time frame. Findings have implications for community and family mental health, and for developing culturally relevant treatments that integrate disciplines.

Keywords: Attachment, Therapeutic camps, Anxiety, Adoption, Foster care.

\section{INTRODUCTION}

Chronic abuse in early childhood, neglect, prolonged and painful medical conditions in infancy, extended separation from nurturing caregivers, or violent family conditions may result in Reactive Attachment Disorder (RAD), which if

\footnotetext{
* Address correspondence to this author at the Cornerstone Therapy and Wellness 639 W Swedesford Rd, Malvern, PA 19355, USA,
} Tel: (610) 616-5935; E-mail: randcoleman@gmail.com 
untreated has deleterious behavioral and psychiatric consequences [1]. Associated behaviors commonly include aggression, hoarding food, lying, stealing and emotional dysregulation while psychiatric concerns include elevated rates of oppositional defiant disorder, conduct disorder, attention deficits and anxiety. More severe periods of neglect are associated with changes in brain development and neural activation [2]. Maladaptive attachment includes nonattachment, disordered attachment and attachment disruption, with nonattachment being the most severe. All forms of damaged attachment imply lack of security or trust in adult caregivers, and all are associated with increased presence of mental health disorders [3].

Treatment approaches nearly all emphasize family or parent-child dyadic components, since the primary issue is security and behavior within a relationship context $[4,5]$. Trust based relationship interventions have been developed to enhance family functioning and to reduce the risk of disrupted adoptions, and show promise for promoting stronger attachment and greater resiliency in relationships [6]. The complex trauma of combined abuse, neglect and loss disrupts attachment development. Best practices for treatment require attachment figures (caregivers) in the therapy process [7]. Parenting a child with severe behavioral problems is exhausting, and when the child rejects parental affection and demonstrates limited emotional warmth, the process can be frustrating and demoralizing. Caregivers must cope with high risk behaviors, such as stealing, lying, elopement and even attempts to abuse siblings. This places the caregivers at risk for stress related emotional disorders and depression, and the child at risk for out-of-home placement. Furthermore, the family system is highly stressed with siblings adversely affected. A camp-like format offers potential for social support of the caregivers in addition to improved emotional-behavioral health of all members.

Intensive camp format for treatment involving the family has been shown to reduce both internalizing and externalizing behaviors in children in foster care who had experienced combined attachment disruption and abuse/neglect and were treatment resistant. In a study by Makela and Vierikko [8], child and parent came for two intensive camps that emphasized parent training and parent-child attachment exercises. The program utilized Theraplay $\AA$, a structured, attachment based approach to family therapy. Therapeutic scheduling included daily individualized parent-child attachment building sessions, group parent consultation and training, and specially planned outings for families to build camaraderie; all occurred over four days of time period. Families were sent home with individualized therapy assignments to be done regularly and staff followed up periodically to provide support. Behavior gains were most dramatic for aggression, rule-breaking, but improvements occurred in social behavior, anxiety, attention and withdrawal/depression. Gains were maintained across domains over a six-month and two year follow-up. Purvis et al. [9] showed that multiple week day-camps for children adopted from orphanage care had a positive impact on behavior and attachment, even when parents had less involvement. Camps provided attachment rich experiences, starting with an attachment ritual for handing off the child from parent to the staff each morning. Sensory rich activities designed by occupational therapists were used for daily group programming, and youth would do relationship building activities with an adult "buddy" (camp counselor) who would model and reinforce prosocial behavior. Behavior checklist ratings showed improvements in thinking process, attention, aggression and attachment behavior. A difference from the Finnish Intensive Theraplay program was limited involvement of caregivers in the camp process.

Despite the promise of the intensive camp format, limited outcome data has been available regarding intensive family camp programs that emphasize attachment and trauma healing. Apart from the studies mentioned above, we were unable to identify any other family based attachment therapy camps that had published data in journal articles.

Attachment specialization program, Families by Design, has held intensive attachment camp programs for families for over 15 years. The camp curriculum was developed with integration of attachment theory, neuroscience, adult and child educational principles, and behavioral psychology. Parent and child emotional factors and behavioral changes were evaluated over a two year period, allowing for outcome assessment of the intervention. Based on clinical reports from the camp staff and case reports from parents, results appeared very promising. Outcome expectations were that youth would show reduced behavior problems, parents would demonstrate less anxiety and stress, and positive attachment behavior would be improved in the context of the parent-child relationship.

\section{METHODS}

\subsection{Setting}

This study examined the immediate and long term effectiveness of week-long intensive therapy family camp in treating children with RAD. Families by Design, Healing Heart Camps are organized around North America, primarily during the summer. The focus of Healing Hearts Camp is to renew, recharge and empower each caregiver (parent, foster 
parent, and grandparent) to increase love and laughter in their home, and for each child to have skills to build honesty, trust and self-control. The camp's goal for families is to find hope and build the skills to be confident to continue with even more healing at home [10]. The camps are week long and involve combinations of activities and interventions designed to change relationship dynamics within families. Key elements of the therapeutic camp include the following:

1. High structure and clear rules to create a sense of safety and boundaries.

2. Parent psychoeducation on topics of attachment, trauma, building emotional connection with children, appropriate and safe discipline and therapeutic strategies.

3. Camp games and activities to promote trust, emotional connection, and emotional safety between family members. Examples include supportive "trust floating" in a pool, creating a family banner with agreed upon values, while caregivers guiding the child on a blindfold obstacle course, and cooperative relay races.

4. Modeling of appropriate discipline by the camp's staff. Punitive actions are avoided in favor of removing the child from a disruptive situation, guiding the child to reflect on why the other person was upset (e.g., "Why was Mom upset?"), guiding the child to generate solutions to fix the relationship break caused by misbehavior or disrespect, and providing support to put the relationship repair into action.

5. High levels of parents' emotional and practical support are provided. This includes consults and counseling for caregivers, assistance with child behavior issues, and facilitating positive interactions. Guidance in providing nurturing relationship repair includes helping caregivers practice warm eye contact, give hugs, and having bonding activities with all family members.

6. Brief and intensive psychotherapeutic interventions are provided on an individualized basis.

7. Self-esteem building and emotional regulation activities are provided in separate group formats for children with RAD and non-diagnosed siblings who often suffer significant stress or secondary trauma.

\subsection{Measures}

To assess the immediate and long-term effectiveness of camp, three tools were used, Randolph Attachment Disorder Questionnaire (RADQ), State Trait Anxiety Inventory (STAI), and a follow-up questionnaire.

The RADQ is a 30 item, Likert-type rating scale [11]. Behavioral items were selected based on their high frequency of occurrence within the population of children treated for attachment disruption. Test-retest reliability was high (.84), and validity research demonstrated that the scale statistically distinguished between attachment disorder and other disruptive disorders. Success in treatment is strongly associated with decrease in total score.

The STAI is a standard instrument for assessing trait and state anxiety [12]. It is composed of 20 items for trait, assessing anxious feelings for the past month, and 20 items for state, assessing current feelings of anxiety. Participants respond to statements such as "I am calm", "I feel secure" on a 4 point scale ("Almost Never to "Almost Always"). Form $\mathrm{Y}$ was used for the adult and form $\mathrm{C}$ was used for children. For the purpose of this study, the STAI was used to compared anxiety trait and states of child and caregiver at the start compared to the end of camp using paired T-test analysis. To determine if there was a correlation between caregiver anxiety and severity of RAD a correlation analysis was also calculated.

The follow-up questionnaire was composed of 7 questions designed to assess the impact of camp on partner relationships, long-term feeling about camp and to determine which aspects of camp were most helpful. The data was presented for informational purposes only. At the end of the online survey, caregivers were given the option to complete a $3^{\text {rd }}$ RADQ which was used for statistical analysis of long term effects.

\subsection{Participants and Procedure}

The study examined data from 72 families attending week-long intensive therapeutic family camps, including eight camps over a two year period (California (CA), Colorado (CO), Tennessee (TN), Florida (FL), Michigan (MI), Oregon (OR), Ohio (OH), and Saskatchewan, Canada (SK)). Prior to coming to camp, families were asked to fill out a RADQ on each child coming to camp who had been diagnosed with RAD; this was to help camp's personnel preparation for families. Upon arrival at camp, families were informed of the study and provided with a written explanation of the study. They completed an informed consent form when agreeing to participation in the research, with the understanding that they could end their participation in the study at any time without a change to the treatment plan. Families that completed a consent form were asked to fill in the appropriate State-Trait Anxiety Scales on the first and the last day of camp. In addition, caregivers were asked to fill out a second RADQ on the last day of camp. The percentage of families 
included in the study was approximately $67 \%$ of those that attended the eight camps. Participation in the study was limited to families with children over 6 years old, RADQ scores above 20, and protocols with complete data sets. Reasons for incomplete data sets included families arriving to camp in total disarray and unable to reliably complete forms, children being noncompliant, families opting not to participate in the study, and end of camp needs. Some families had more than one child with RAD resulting in data collected on a total of 131 RAD children $(\mathrm{CO} N=18 ; \mathrm{FL}$ $\mathrm{N}=23$; $\mathrm{CA} \mathrm{N}=11$; $\mathrm{TN} \mathrm{N}=13$; OR N=21; MI N=15; $\mathrm{SK} \mathrm{N}=12$; OH N=18. Six months following the last camp, Families by Design sent out individual invitations with unique identification codes to all participants with a link to an electronic follow-up survey. Thirty-six of the 72 families responded, providing data for 55 children. The follow-up period reported ranges from 6 to 30 months post camp.

All statistical analysis was carried out by researchers at Cabrini University, none of whom had attended any of the therapeutic camps to avoid being biased by personal camp exposure. All collected data was mailed to Cabrini University with codes to preserve anonymity; no additional background information on individual families was provided to the researchers.

\subsubsection{RADQ Data Analysis}

Children with RAD had been diagnosed prior to camp by an independent mental health professional in their own community. For this study, RADQ results were used solely to assess behavioral severity levels. All RADQ scores reported are the raw RADQ score minus 30 baseline points per protocol developed by Elizabeth Randolph [11]. A severity rating system for the study was developed to classify children into clinical categories for analysis. Mild RAD was $20-45$ points, moderate RAD was $46-60$ points, severe RAD was $61-75$ points and very severe RAD was $75+$ points. Based on severity ranking, two-tailed paired student T-tests were run to measure changes before camp to the end of camp and before camp to the follow-up point. Cohen's $d$ was used to measure the effect . RADQ was also used to identify which behaviors were identified as the most severe.

Previous research by Elizabeth Randolph [11] suggested that only RADQs filled out by the primary caregiver should be considered valid. Because it was not always clear from the paperwork who was the primary caregiver, there was an initial analysis to determine if there was a statistical difference between RADQ scores obtained by "mom" $v s$. "dad" vs. "other". There was no statistical significant difference $(\mathrm{t}=-0.047 ; \mathrm{p}=0.5)$. Where possible, the RADQ filled out by the identified primary caregiver was utilized, but in cases where there were multiple RADQ questionnaires completed, the first one entered was used for the purpose of analysis.

\subsubsection{STAI Data Analysis}

The complete STAI was administered to all participants on the first day of camp. The "state" portion was readministered on the last day of camp. The "trait" form was used to establish a baseline anxiety assessment while the "state" form was utilized to measure the current feelings of anxiety upon arrival at camp and at the end of camp. Twotailed paired t-tests were used to determine significant difference in state anxiety at the beginning compared to the end of camp. A correlation coefficient was also calculated to assess correlations between caregiver anxiety and severity of RAD.

\subsection{Questionnaire Analysis}

The answers in the follow-up questionnaire were quantified to determine the overall effectiveness of camp and ranking of effectiveness of different parts of camp. Additional statistical analysis was not completed.

\section{RESULTS}

\subsection{Who Comes to Camp and What Are the Key Issues?}

To determine who is utilizing this type of therapeutic approach, the RADQ scores were used to determine a general profile of families availing themselves of this therapeutic approach. Contrary to general impressions, camps served the full spectrum of children with RAD. While each camp was unique in terms of number of families, size of families, facilities and setting, there was general consistency in overall distribution of children with severity of RAD that ran the spectrum from mild to very severe (Table 1). 
Table 1. Percentage of children at each RAD severity ranking.

\begin{tabular}{|c|c|c|c|c|}
\hline CAMP & Mild & Moderate & Severe & Very Severe \\
\hline CO & $6 \%$ & $11 \%$ & $33 \%$ & $50 \%$ \\
\hline FL & $22 \%$ & $13 \%$ & $35 \%$ & $30 \%$ \\
\hline CA & $9 \%$ & $36 \%$ & $9 \%$ & $45 \%$ \\
\hline TN & $15 \%$ & $15 \%$ & $31 \%$ & $38 \%$ \\
\hline OR & $14 \%$ & $29 \%$ & $29 \%$ & $29 \%$ \\
\hline MI & $0 \%$ & $40 \%$ & $7 \%$ & $53 \%$ \\
\hline SK & $25 \%$ & $8 \%$ & $16 \%$ & $50 \%$ \\
\hline OH & $17 \%$ & $22 \%$ & $22 \%$ & $39 \%$ \\
\hline All Combined & $19 \%$ & $22 \%$ & $24 \%$ & $41 \%$ \\
\hline
\end{tabular}

In addition to the overall profile of severity, the initial RADQ was also used to examine which behavior caregivers were perceiving as the most problematic. Table (2) presents the summary of each RADQ item and Fig. (1) shows the average profile of the children based on severity of individual items on the RADQ. There was remarkable consistency among the severity categories of RAD. This lends support to the idea that attachment disorder falls on a continuum. One caveat to this was a few areas of notable discrepancy in the profile of Mild RAD compared to moderate through very severe. Specifically, children diagnosed with Mild RAD had better eye contact tolerance than predicted by the other profiles and were rated as more impaired in keeping friends as compared to the children with being very severe RAD.

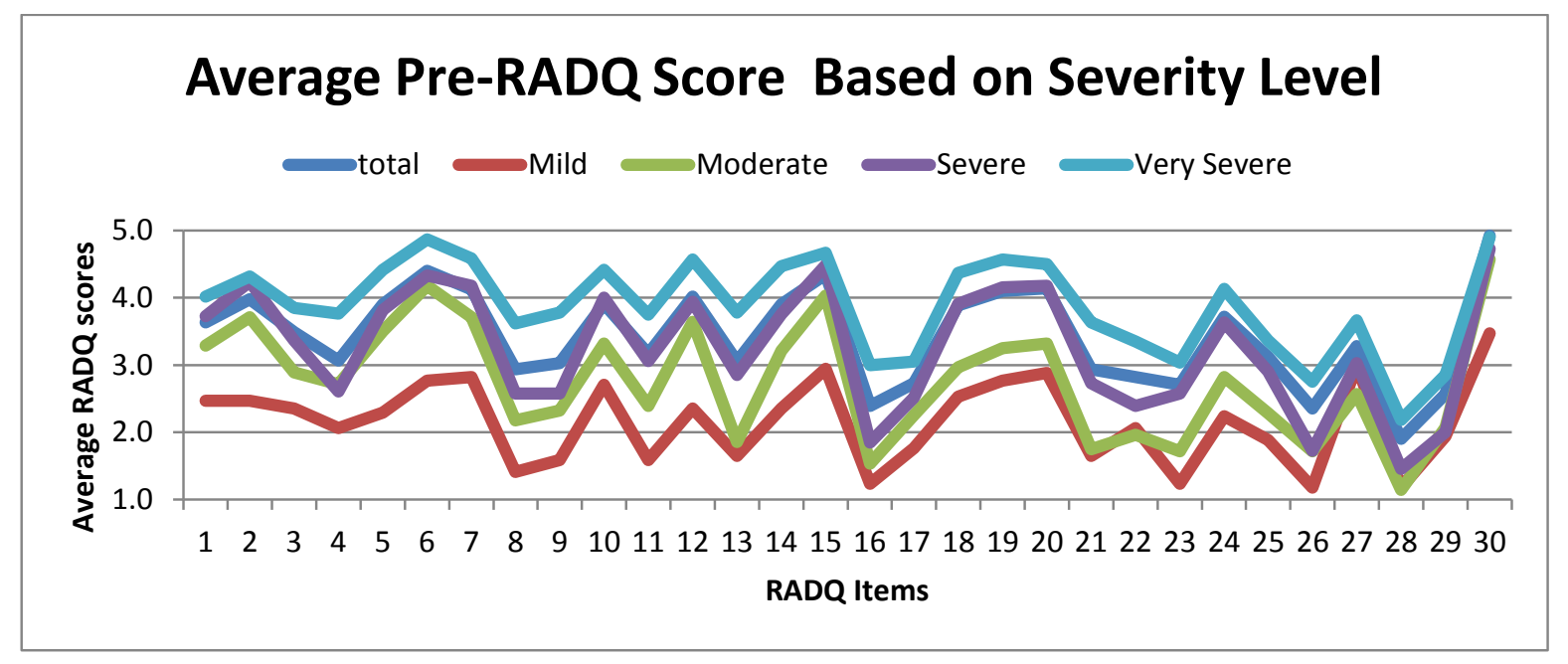

Fig. (1). The figure above is a profile of average RADQ items for each severity rank. 1=normal behavior; $5=$ most severe behavior.

Table 2. Summary of RADQ items.

\begin{tabular}{|l|l|}
\hline 1. Acts overly cute \& charming & 16. Tries for sympathy by claiming abuse \\
\hline 2, Trouble making eye contact & 17. Shakes off pain, refusing comfort \\
\hline 3, Overly friendly with strangers & 18. Sneaks things without permission \\
\hline 4. Pushes away or becomes stiff when hugged & 19. Pathological liar \\
\hline 5. Argues for long periods & 20. Very bossy with other children \\
\hline 6. Tremendous need for control & 21. Sneaks food \\
\hline 7. Acts amazingly innocent when caught & 22. Can't keep friends \\
\hline 8. Does dangerous things & 23. Throws temper tantrums \\
\hline 9.Deliberately breaks things & 24. Non-stop chatter \\
\hline 10. Does not appear to feel age-appropriate guilt & 25. Accident prone \\
\hline 11. Teases, hurts or cruel to other children & 26. Hurts animals \\
\hline 12. Impulsive & 27. Under achieves in school \\
\hline 13 Steals/ shows up with things that do not belong to them & 28. Sets fires \\
\hline 14. Demands instead of asking & 29. Prefers violent shows \\
\hline 15. Does not seem to learn from misbehavior & 30.Early childhood trauma \\
\hline
\end{tabular}


What specific behaviors do children display the most are of particular interest to therapeutic personnel. The top 10 items were calculated for each severity grouping. This analysis found relatively high consistency regarding which behaviors are perceived as the most problematic in mild to very severe RAD children. In all the levels of severity among the top 10 perceived problematic behaviors, 5 behaviors were consistently reported: "Very bossy with other children and adults"; "Does not learn from mistakes \& misbehavior, no matter the consequence"; "Tremendous control over everything, very upset if things don't go his/her way"; "Acts amazingly innocent when caught doing something wrong"; and "Does not seem to feel age appropriate guilt".

\subsection{State/Trait Anxiety Index (STAI) Data}

The STAI was assessed as part of understanding characteristics of families that utilize this type of intensive intervention. The caregivers had an average trait score of $44.7 \pm 9.4$. Based on clinical use of the measure, this placed the group as a whole at moderate to severe level of anxiety. There was no significant difference between the camps, nor was there a correlation between the caregiver trait score and child RADQ score, reinforcing the knowledge that how RAD caregivers feel is based on their own experience and cannot be predetermined by practitioners based on severity of RAD ( $\mathrm{r}=0.056$; no correlation). Very interestingly, caregiver trait (baseline) vs. initial state (immediate) score decreased statistically significantly $(\mathrm{p}<0.001)$, suggesting that just coming to the camp had significant impact on their sense of support and hope (Table 3 ).

Table 3. Effect of arriving at camp on state anxiety compared to Trait Anxiety Index.

\begin{tabular}{|c|c|c|c|c|c|c|}
\hline & Trait & Pre State & p value & Effect size & \multicolumn{2}{|c|}{ Cohen's $\boldsymbol{\text { }}$} \\
\hline Caregiver & $44.7 \pm 9.4$ & $42.1 \pm 12.1$ & $<0.001$ & 0.118 & 0.23 & small \\
\hline RAD Child (CO \& FL) & $46.7 \pm 10$ & $37.3 \pm 12$ & $<0.001$ & 0.39 & 0.85 & large \\
\hline RAD Child (CA \& TN) & $36.3 \pm 7$ & $31 \pm 8$ & $<0.001$ & 0.33 & 0.7 & medium \\
\hline
\end{tabular}

Likewise RAD identified children exhibited a similar result. The study was refined over the course of the 8 camps, resulting in a switch to a child version of the State-Trait Anxiety scale for the CA and TN camps, therefore the data were separated for the camps based on the scale used. Both the scales showed the same effect, a decrease in anxiety levels. This suggests that just arriving to the camp reduced the daily stress felt by these children.

\subsection{Immediate Impact of Camp}

Overall RADQ analysis of all 131 subjects across all camps and severity ratings showed that there was a significant change in thepre-post RADQ scores $(\mathrm{p}<0.001)$ from the first day of camp to the last. Effect size was 0.568; Cohen's $d$ (measure of significance of effect size) was calculated to be 1.379 indicating a large effect size. The data clearly indicates that camp had an immediate effect on reducing perceived overt RAD associated behaviors.

All camps exhibited a significant decrease in RADQ scores from pre to post with a $p$ value $<0.01$. Camps collectively had a t stat $=9.15 \mathrm{E}-31, \mathrm{p}$ value $<0.001$ and effect size and Cohen's d were also calculated for each camp and for each RAD severity group Tables (4 and 5). All camps exhibited a large effect size suggesting consistency among the camps. MI had the smallest calculated effect size; however, MI also had the largest percentage of very severe RAD children and thus this may account for the smaller effect size as these children are most difficult and may need additional attachment based therapy beyond what camp can offer. As seen in Table (5), when the immediate effect of camp was evaluated based on RAD severity, all severity levels had a large effect size, suggesting that children with any severity of RAD can be helped through this intensive therapeutic approach. Those individuals with RAD ranking of severe showed the greatest improvement during the week at camp. Those individuals with mild RAD, as determined by RADQ scores, did exhibit a large effect size, but smaller as compared to the severe group. This could be due to the fact that they had much less room for improvement.

Table 4. Pre/post total RADQ scores based on location.

\begin{tabular}{|c|c|c|c|c|c|c|}
\hline Location & Pre -Average & Post - Average & p-value & Effect Size & \multicolumn{2}{|c|}{ Cohen's $\boldsymbol{L}$} \\
\hline CO & $74.7 \pm 18.5$ & $32.9 \pm 16.6$ & $<0.001$ & 0.765 & 2.37 & Large \\
\hline FL & $68.3 \pm 18.5$ & $44.7 \pm 16.6$ & $<0.001$ & 0.493 & 1.134 & Large \\
\hline CA & $66.5 \pm 17.5$ & $46.5 \pm 19.5$ & $<0.001$ & 0.474 & 1.078 & Large \\
\hline TN & $70.6 \pm 21.1$ & $47.8 \pm 22.4$ & $<0.01$ & 0.461 & 1.039 & Large \\
\hline OR & $62.0 \pm 17.3$ & $26.5 \pm 13.9$ & $<0.001$ & 0.756 & 2.311 & Large \\
\hline
\end{tabular}


(Table 4) contd.....

\begin{tabular}{|c|c|c|c|c|c|c|}
\hline Location & Pre-Average & Post - Average & p-value & Effect Size & \multicolumn{2}{|c|}{ Cohen's $d$} \\
\hline MI & $74.2 \pm 20.9$ & $53.1 \pm 30.0$ & $<0.001$ & 0.376 & 0.811 & Large \\
\hline SK & $67.1 \pm 26.2$ & $37.8 \pm 19.5$ & $<0.001$ & 0.587 & 1.449 & Large \\
\hline OH & $66.1 \pm 24.5$ & $36.0 \pm 27.5$ & $<0.001$ & 0.506 & 1.174 & Large \\
\hline Combined & $68.5 \pm 20.8$ & $39.5 \pm 22.6$ & $<0.001$ & 0.568 & 1.379 & Large \\
\hline
\end{tabular}

Table 5. Pre/post RADQ based on severity rank.

\begin{tabular}{|c|c|c|c|c|c|c|}
\hline Severity & Pre -Average & Post - Average & p-value & Effect Size & \multicolumn{2}{|c|}{ Cohen's $\boldsymbol{l}$} \\
\hline Mild & $33.9 \pm 7.7$ & $23.2 \pm 14.0$ & $<0.01$ & 0.445 & 0.993 & Large \\
\hline Moderate & $52.4 \pm 4.6$ & $34.0 \pm 17.5$ & $<0.001$ & 0.583 & 1.486 & Large \\
\hline Severe & $68.1 \pm 3.6$ & $37.3 \pm 14.5$ & $<0.001$ & 0.833 & 3.01 & Large \\
\hline Very Severe & $88.3 \pm 10.4$ & $49.7 \pm 25.9$ & $<0.001$ & 0.704 & 1.98 & Large \\
\hline
\end{tabular}

The RADQ was not designed for use in a camp setting, and therefore, investigators identified the specific items they believed could be addressed at camp and carried out the analysis on just these items. The specific items assessed are listed in Table (6) along with the average RADQ rating pre and post for each of the critical items based on severity rating. All targeted behaviors showed improvement (lower score) across the week of camp. In the mild group there was a statistical improvement in 5 of the 15 behaviors. In the moderate severity group, there was statistically significant improvements in the majority of behaviors except "shaking off pain", "temper tantrums", "chattering" and "accident prone". In the severe and very severe groups, all targeted behaviors showed a statistically significant improvement. Effect size was also calculated for the critical items Table (7). There was a significant effect observed on the critical items based on severity rank as measured using Cohen's d. The most significant effect on the critical items was observed in the very severe rank. This is slightly different from the trend observed when all items of the RADQ were included.

Table 6. Camp determined list of critical items from the RADQ.

\begin{tabular}{|c|c|c|c|c|c|c|c|c|}
\hline & $\begin{array}{l}\text { Mi. } \\
\text { pre }\end{array}$ & $\begin{array}{l}\text { Mi. } \\
\text { post }\end{array}$ & $\begin{array}{l}\text { Mo. } \\
\text { pre }\end{array}$ & $\begin{array}{l}\text { Mo. } \\
\text { post }\end{array}$ & $\begin{array}{l}\text { Sev. } \\
\text { pre }\end{array}$ & $\begin{array}{l}\text { Sev. } \\
\text { post }\end{array}$ & $\begin{array}{l}\text { VS } \\
\text { Pre } \\
\end{array}$ & $\begin{array}{c}\text { VS } \\
\text { post }\end{array}$ \\
\hline $\begin{array}{l}\text { 1: Overly } \\
\text { Cute }\end{array}$ & 2.4 & 2.2 & 3.2 & $3.1 *$ & 3.8 & $2.8 * * *$ & 4.1 & $3.2 * * *$ \\
\hline \begin{tabular}{|l|} 
2: Trouble \\
making Eye \\
contact
\end{tabular} & 2.4 & 2.4 & 3.7 & $2.9 * *$ & 4.3 & $2.9 * * *$ & 4.4 & $3.2 * * *$ \\
\hline \begin{tabular}{|l|} 
4: Pushes \\
away when I \\
try to hug
\end{tabular} & 2.0 & 1.6 & 2.7 & $2.1^{*}$ & 2.6 & $1.6 * * *$ & 3.8 & $2.5 * * *$ \\
\hline \begin{tabular}{|l|} 
5: Argues \\
for long \\
periods of \\
time \\
\end{tabular} & 2.2 & 1.8 & 3.4 & $1.9 * * *$ & 3.9 & $2.2 * * *$ & 4.5 & $2.7 * * *$ \\
\hline \begin{tabular}{|l|}
$6:$ \\
Tremendous \\
need for \\
Control \\
\end{tabular} & 2.7 & 2.2 & 4.0 & $2.8 * * *$ & 4.5 & $2.7 * * *$ & 4.9 & $3.1 * * *$ \\
\hline $\begin{array}{l}\text { 7: Acts } \\
\text { innocent } \\
\text { when } \\
\text { caught } \\
\text { doing } \\
\text { something } \\
\text { wrong } \\
\end{array}$ & 2.8 & 1.8 & 3.7 & $2.2 * * *$ & 4.2 & $2.6 * * *$ & 4.6 & $3.2 * * *$ \\
\hline $\begin{array}{l}\text { 10: Does not } \\
\text { seem to feel } \\
\text { guilt }\end{array}$ & 2.8 & $1.9 *$ & 3.4 & $2.7^{*}$ & 3.9 & $2.8 * * *$ & 4.5 & $3.4 * * *$ \\
\hline
\end{tabular}




\begin{tabular}{|c|c|c|c|c|c|c|c|c|}
\hline & $\begin{array}{l}\text { Mi. } \\
\text { pre }\end{array}$ & $\begin{array}{l}\text { Mi. } \\
\text { post }\end{array}$ & $\begin{array}{l}\text { Mo. } \\
\text { pre }\end{array}$ & $\begin{array}{l}\text { Mo. } \\
\text { post }\end{array}$ & $\begin{array}{l}\text { Sev. } \\
\text { pre }\end{array}$ & $\begin{array}{l}\text { Sev. } \\
\text { post }\end{array}$ & $\begin{array}{l}\text { VS } \\
\text { Pre }\end{array}$ & $\begin{array}{c}\text { VS } \\
\text { post }\end{array}$ \\
\hline \begin{tabular}{|l|} 
12: Seems \\
unable to \\
stop himself \\
from doing \\
things \\
impulsively \\
\end{tabular} & 2.4 & $1.6^{*}$ & 3.6 & $2.4 * * *$ & 4.1 & $2.8 * * *$ & 4.6 & $3.0 * * *$ \\
\hline $\begin{array}{l}\text { 14: } \\
\text { Demands } \\
\text { instead of } \\
\text { asking of } \\
\text { asking }\end{array}$ & 2.3 & 1.5 & 3.2 & $2.3 * * *$ & 3.8 & $2.7 * * *$ & 4.5 & $3.0 * * *$ \\
\hline $\begin{array}{l}\text { 17: Shakes } \\
\text { off pain, } \\
\text { accepts no } \\
\text { comfort }\end{array}$ & 1.8 & 1.4 & 2.2 & 2.0 & 2.6 & $1.7 * *$ & 3.1 & $2.5 * * *$ \\
\hline $\begin{array}{l}\text { 18: Sneaks } \\
\text { w/o } \\
\text { permission }\end{array}$ & 2.5 & $1.4 * *$ & 3.1 & $1.9 * * *$ & 4.0 & $2.4 * * *$ & 4.4 & $2.5 * * *$ \\
\hline \begin{tabular}{l|}
$19:$ \\
Pathological \\
liar
\end{tabular} & 2.7 & $1.7 *$ & 3.3 & $2.3 * * *$ & 4.2 & $2.6 * * *$ & 4.6 & $3.0 * * *$ \\
\hline $\begin{array}{l}\text { 23: Temper } \\
\text { Tantrums } \\
\text { that last for } \\
2 \text { hour }+\end{array}$ & 1.2 & 1.0 & 1.6 & 1.2 & 2.5 & $1.5 * * *$ & 3.1 & $2.1 * * *$ \\
\hline $\begin{array}{l}\text { 24: Chatters } \\
\text { non-stop, } \\
\text { asks } \\
\text { repeated } \\
\text { questions, } \\
\text { other speech } \\
\text { oddities }\end{array}$ & 2.3 & $1.5^{*}$ & 2.7 & 2.0 & 3.6 & $2.3 * * *$ & 4.3 & $3.0 * * *$ \\
\hline $\begin{array}{l}\text { 25: Accident } \\
\text { prone; }\end{array}$ & 1.8 & 1.3 & 2.2 & 2.2 & 2.9 & $1.7 * * *$ & 3.4 & $2.3 * * *$ \\
\hline
\end{tabular}

Mi.=mild; Mo. $=$ Moderate; Sev $=$ severe; VS=very severe; ${ }^{*} \mathbf{p}<. \mathbf{0 5} ; * * \mathbf{p}<. \mathbf{0 1} ; * * * \mathbf{p}<.001$ For reference $1=$ normal behavior; $5=$ most severe behavior.

Table 7. Immediate effect size for targeted RADQ items based on severity.

\begin{tabular}{|c|c|c|c|c|c|c|}
\hline Severity & Pre Average & Post Average & P value & Effect size & \multicolumn{2}{|c|}{ Cohen's d } \\
\hline Mild & $19.2 \pm 6.7$ & $10.3 \pm 7.7$ & $<0.01$ & 0.52 & 1.23 & Large \\
\hline Moderate & $31.1 \pm 4.1$ & $19.0 \pm 10.1$ & $<0.001$ & 0.62 & 1.58 & Large \\
\hline Severe & $39.6 \pm 5.5$ & $20.1 \pm 12.1$ & $<0.001$ & 0.49 & 1.15 & Large \\
\hline Very Severe & $47.5 \pm 5.3$ & $27.4 \pm 14.0$ & $<0.001$ & 0.68 & 1.89 & Large \\
\hline
\end{tabular}

In addition to examining critical items on the RADQ, state trait scores were also evaluated Table (8). Reduction on this anxiety scale was significant for all participants. The calculated effect size and Cohen's d showed a medium effect.

\subsection{Long Term Impact of Camp}

The long-term follow-up data (6- 30 months) suggests that the effects observed at the camp were long lasting. Follow-up questionaries were sent 6 to 30 months post camp. 36 of the original 72 families responded to the follow-up questionnaire providing data on 55 children. $98 \%$ of those responding still felt like the time and expense of camp were worth it and would recommend this type of intervention to other families regardless of RAD severity.

In response to how the RAD children's overall behavior was before the camp, $42 \%$ of the respondents reported that behavior ultimately continued to improve compared to behaviors at the end of camp. While 40\% reported ups and down in behavior but generally the positive changes observed at the camp sustained. Only $9 \%$ of the RAD children were reported to have worsened since the camp was started and 4 children from the follow-up group had been moved to other homes. 
Table 8. Effect of camp on state anxiety compared to Trait Anxiety Index.

\begin{tabular}{|c|c|c|c|c|c|c|}
\hline & Trait & Post State & P value & Effect Size & \multicolumn{2}{|c|}{ Cohen's d } \\
\hline Caregiver & $44.7 \pm 9.4$ & $\mathbf{3 8 . 6 1} \pm \mathbf{1 1 . 3}$ & $\mathbf{p}<\mathbf{0 . 0 0 1}$ & $\mathbf{0 . 5 9}$ & $\mathbf{0 . 3}$ & Medium \\
\hline RAD Child (CO \& FL) & $\mathbf{4 6 . 7 \pm 1 0}$ & $\mathbf{3 4 . 3 3} \pm 9.3$ & $\mathbf{p}<\mathbf{0 . 0 0 1}$ & $\mathbf{1 . 2 8}$ & $\mathbf{0 . 5}$ & Medium \\
\hline RAD Child (CA \& TN) & $\mathbf{3 6 . 3} \pm 7$ & $\mathbf{3 0 . 0 7} \pm \mathbf{8 . 2}$ & $\mathbf{p}<\mathbf{0 . 0 0 1}$ & $\mathbf{0 . 8 1}$ & $\mathbf{0 . 4}$ & Medium \\
\hline
\end{tabular}

These behavioral observations were further analyzed through follow-up RADQ scores, which were completed for 40 of the original 131 children. All statistical data presented in this section includes only data from the specific 40 children. Overall RADQ analysis Table (9) of the 40 subjects across all camps and severity ratings showed that there was a statistically significant change in the Follow-up RADQ scores $(p<0.001)$. The effect size was 0.337 ; Cohen's $d$ was 0.716 indicating a sustained medium effect size following the camp. Table (8) summarizes the RADQ data based on severity rating. With the exception of the mild group, all children appeared to have a sustained improvement in RADQ symptoms before the camp. In contrast, the averaged mild group data appeared to suggest a worsening of RADQ symptoms post camp; however, the follow-up includes data from only 4 of the original 16 and 1 child, in particular, reported significant worsening of symptoms which significantly impacted the overall averages of this group.

Table 9. Pre/follow-up total RADQ based on severity.

\begin{tabular}{|c|c|c|c|c|c|c|c|}
\hline Severity & N & Pre-Average^ $^{\wedge}$ & Follow-up Average & P value & Effect Size & \multicolumn{2}{c|}{ Cohen's $\boldsymbol{d}$} \\
\hline Mild & 4 & 29.75 & 36 & 0.196 & 0.213 & 0.435 & Small \\
\hline Moderate & 10 & 53.1 & 34.5 & 0.02 & 0.506 & 1.172 & Large \\
\hline Severe & 10 & 68.3 & 47.2 & 0.065 & 0.0429 & 0.949 & Large \\
\hline Very Severe & 16 & 90 & 67.25 & 0.003 & 0.477 & 1.084 & Large \\
\hline Combined & 40 & 69.3 & 50.9 & $<0.001$ & 0.337 & 0.716 & Medium \\
\hline
\end{tabular}

Average includes only Children with both a pre-camp and follow-up RADQ rating

Camp was designed to specifically target certain behaviors identified in the RADQ with the expectation of a globalized improvement. Additional analyses was done on those specific behaviors observed in the camp. Table (10) is a summary of the findings. Overall the targeted behaviors continued to show improvements after families returned home from the camp. Compared to immediate RADQ scores for the targeted behaviors, there were fewer statistically significant differences, however, the calculated effect size was still large for all levels of severity (Table 11).

Table 10. Long term effect size for targeted RADQ items based on severity.

\begin{tabular}{|c|c|c|c|c|c|c|}
\hline Severity & $\begin{array}{c}\text { Pre Camp } \\
\text { Average (stdev) }\end{array}$ & $\begin{array}{c}\text { Follow-up } \\
\text { Average (stdev) }\end{array}$ & P value & Effect size & \multicolumn{2}{|c|}{ Cohen's d } \\
\hline Mild & $17( \pm 4.2)$ & $19.5( \pm 9.1)$ & 0.5 & 0.17 & 0.35 & Small \\
\hline Moderate & $30.7( \pm 5.3)$ & $15.3( \pm 11.2)$ & $<0.001$ & 0.66 & 1.76 & Large \\
\hline Severe & $37.4( \pm 9.2)$ & $20.0( \pm 12.5)$ & $<0.05$ & 0.62 & 1.58 & Large \\
\hline Very Severe & $47.9( \pm 5.6)$ & $28.9( \pm 12.2)$ & $<0.01$ & 0.71 & 2 & Large \\
\hline
\end{tabular}

In addition to overt behavioral changes, the follow-up questionnaire also examined impacts on changes in family relationships. $68 \%$ of the respondents reported improved connection between the primary caregiver and child following the camp and only 3 caregivers felt that the camp has worsened the sense of connection between the primary caregiver and their child; and in 2 of the cases, the child had since been moved to a different home. Overall the camp seemed to have a positive impact on caregivers coming together as a team. The majority of families, $68 \%$, that came to the camp with more than one caregiver reported that the sense of working as a team in parenting the child had improved . There was a small portion, $14 \%$ that reported that after an initial increase in the sense of team work, it did not sustain and they returned to the status quo they had before the camp.

Table 11. Follow-up camp determined critical items averages by severity grouping.

\begin{tabular}{|l|c|c|c|c|c|c|c|c|}
\hline & $\begin{array}{c}\text { Mi. } \\
\text { pre }\end{array}$ & $\begin{array}{c}\text { Mi } \\
\text { L.T. }\end{array}$ & $\begin{array}{c}\text { Mo. } \\
\text { pre }\end{array}$ & $\begin{array}{c}\text { Mo. } \\
\text { L.T. }\end{array}$ & $\begin{array}{c}\text { Sev. } \\
\text { pre }\end{array}$ & $\begin{array}{c}\text { Sev. } \\
\text { L.T. }\end{array}$ & $\begin{array}{c}\text { VS } \\
\text { pre }\end{array}$ & $\begin{array}{c}\text { VS } \\
\text { L.T. }\end{array}$ \\
\hline 1: Overly Cute & 2.4 & 2.3 & 3.2 & $1.3^{*}$ & 3.8 & 2.9 & 4.1 & 3.6 \\
\hline 2: Trouble making Eye contact & 2.4 & 2.4 & 3.7 & $1.3^{*}$ & 4.3 & 2.7 & 4.4 & 3.9 \\
\hline 4: Pushes away when I try to hug & 2.0 & 1.8 & 2.7 & 1.3 & 2.5 & 1.9 & 3.8 & 3.0 \\
\hline
\end{tabular}




\begin{tabular}{|c|c|c|c|c|c|c|c|c|}
\hline & $\begin{array}{l}\text { Mi. } \\
\text { pre }\end{array}$ & \begin{tabular}{r|} 
Mi \\
L.T.
\end{tabular} & $\begin{array}{l}\text { Mo. } \\
\text { pre }\end{array}$ & $\begin{array}{l}\text { Mo. } \\
\text { L.T. }\end{array}$ & \begin{tabular}{|c|} 
Sev. \\
pre
\end{tabular} & $\begin{array}{l}\text { Sev. } \\
\text { L.T. }\end{array}$ & $\begin{array}{l}\text { VS } \\
\text { pre }\end{array}$ & \begin{tabular}{|l|} 
VS \\
L.T. \\
\end{tabular} \\
\hline 5: Argues for long periods of time & 2.2 & 2.0 & 3.4 & 1.0 & 3.9 & 2.6 & 4.5 & 4.0 \\
\hline 6: Tremendous need for control & 2.7 & 2.4 & 4.0 & $1.7 * * *$ & 4.5 & 3.1 & 4.9 & $4.2^{* *}$ \\
\hline 7: Acts innocent when caught doing something wrong & 2.8 & 2.3 & 3.7 & $2.0 * * *$ & 4.2 & 2.4 & 4.6 & $4.0^{*}$ \\
\hline 10: Does not seem to feel guilt & 2.8 & 2.3 & 3.4 & 1.7 & 3.9 & 2.6 & 4.5 & $3.8^{*}$ \\
\hline 12: Seems unable to stop him/herself from doing things Impulsively & 2.4 & 2.0 & 3.6 & $1.7^{*}$ & 4.1 & 2.7 & 4.5 & 4.1* \\
\hline $\begin{array}{l}\text { 14: Demands instead of asking } \\
\text { of asking }\end{array}$ & 2.3 & 1.9 & 3.2 & 1.3 & 3.8 & 1.7 & 4.5 & $3.3^{* *}$ \\
\hline $\begin{array}{l}\text { 17: Shakes off pain, } \\
\text { accepts no comfort }\end{array}$ & 1.8 & $1.6 *$ & 2.2 & $1.3^{*}$ & 2.5 & 1.9 & 3.1 & 2.6 \\
\hline 18: Sneaks w/o permission & 2.5 & 2.0 & 3.1 & $1.0^{*}$ & 4.0 & 3.4 & 4.4 & $3.7 *$ \\
\hline 19: Pathological liar & 2.7 & $2.2 *$ & 3.3 & $1.0^{*}$ & 4.2 & 2.7 & 4.6 & $3.7 * *$ \\
\hline 23: Temper Tantrums that last for 2 hour + & 1.2 & 1.1 & 1.6 & 1.7 & 2.5 & 2.7 & 3.1 & $4.1^{*}$ \\
\hline 24: Chatters non-stop, asks repeated questions, other speech oddities & 2.3 & 1.9 & 2.7 & $1.0 * * *$ & 3.5 & 2.3 & 4.2 & $3.0^{* * * *}$ \\
\hline 25: Accident prone; & 1.8 & 1.5 & 2.2 & 1.7 & 2.9 & 1.1 & 3.4 & $2.1 * *$ \\
\hline
\end{tabular}

The final portion of the follow-up questionnaire asked families to reflect back on 8 components that every camp incorporates and rate them on a scale of 4 point scale ranging from extremely helpful to discouraging. Table (12 summarizes the findings. The top 3 components that families found most helpful, all centered around the direct training including opportunities to observe the techniques being put into practice by staff and being able to practice with support. This was closely followed by the help reieved by meeting other caregivers with similar children. Networking post camp was felt to be the least effective.

Table 12. Effectiveness of camp components.

\begin{tabular}{|l|c|}
\hline \multicolumn{1}{|c|}{ Camp Component } & Rating \\
\hline Direct Parent training & 3.9 \\
\hline Learning by watching staff work with my child & 3.7 \\
\hline Hands-on practice of techniques with staff support & 3.7 \\
\hline Meeting other caregivers with similar children & 3.6 \\
\hline Trust building/ family bonding activities & 3.5 \\
\hline Ideas gained from sharing and listening to other caregivers/staff & 3.4 \\
\hline Partner bonding & 3.1 \\
\hline Networking for post camp support & 3 \\
\hline
\end{tabular}

Scale: 4=Extremely helpful; $3=$ Helpful; $2=$ Made no difference; 1 = Discouraging

\section{DISCUSSION}

Overall this study provides additional evidence to support the benefits of intensive family camp therapeutic intervention for the treatment of Reactive Attachment Disorder. The findings in this study extend those by Makela and Vierikko [8] to include the efficacy of this type of treatment for intact families in addition to long term foster care. The specific intensive therapeutic family camps facilitated by Families by Design provided positive outcomes in reducing negative behaviors associated with RAD and increased connection between caregivers and caregiver-child.

Who comes to the camp and what are the key issues?

Defining who comes to camp can serve to better shape activities and focus energy on helping both caregivers and children to heal and find hope and support. This study demonstrates that while the majority of families are bringing children with severe to very severe RAD, families with children with less severe RAD symptoms also come to the camp. This suggests that while primary focus should be on meeting the needs of the children with severe RAD, there needs to be an acknowledgement that some of the attendees are not as severe and may have other issues, such as peer relationships and school issues which need to be addressed.

While the overall profile based on the RADQ appears to be on a continuum of severity with similar behavioral profiles, there were discrepancies in the level of difficulty with eye contact and maintaining friendships. Difficulty with eye contact differed across severity levels. Eye contact is an important social behavior related to the ability to accept nurture from a caretaker and also related to feelings of shame and guilt. One hypothesis is that the children in the Mild group are able to tolerate significantly higher levels of affection (including warm eye contact) than children with more 
severe RAD. A second hypothesis is that they have less sense of personal shame, or conversely, better self-esteem. Inability to keep friends may be a function of their actually having generally better behavior than children in the more severe categories. Children with very severe behavior are often exceptionally restricted in their social opportunities, therefore, having no friends to lose. Children with milder behavior problems are more likely to be programmed in regular education and typical after-care or recreational programs, providing caregivers with more opportunities to observe them alienating peers. When compared to typically developing peers, the contrast can become quite glaring.

Quality of attachment has been directly related to peer relationship [13, 14]. Peer relationships are to some degree a barometer of the many components of attachment behavior, including conscience development, integrity, empathy, social problem solving, mediation of conflict and management of emotions. These are all domains of social and emotional intelligence [15].These skills are at such deficit levels for many children with long-standing attachment and behavior deficits that remediation is needed for them to develop these skills

This study also provides important information on those behaviors families are reporting as the most troublesome so they can be specifically targeted. The profile for this analysis was based on caregiver answers to the RADQ, a perception based scale. A Likert-type scale such as the RADQ is not a direct, observational measure of behavioral frequency and should not be interpreted as being representative of which behaviors actually happen with greatest frequency. However, it does reflect parent perception of which behaviors are the most frequent, intense, or severe. Clinical experience and traditional validity studies have found that the RADQ scores generally reflect severity of behavioral difficulty presented by a child [11]. This would help to explain some of the differences between severity profiles. Children diagnosed with RAD are commonly described as controlling and bossy, failing to learn from mistakes or consequences, lacking a sense of guilt and quick to prevaricate. One item was notable for appearing in the top 10 most problematic behaviors for the mild group that did not appear in the top 10 of any other severity rank - "Does not do as well in school as he/she could with even a little more effort". As stated earlier because the RADQ is a perception based scale, this could reflect a change in the parent focus. Once problematic behaviors are decreased at home, school becomes more of a focus.

Across the spectrum, striving for control predominates, a finding is consistent with the lack of trust in caretakers or environmental safety. Conversely, behaviors absent in the top ten is also noteworthy. In this study, certain destructive behaviors often associated with RAD, i.e., cruelty to animals, breaking things and prolonged tantrums, did not make the top ten list. While these behaviors do occur with these children, the frequency relative to other behaviors is perceived as lower.

The STAI data supports research by Wimmer, Vonk and Reeves [16], who found that Attachment Therapy provided to adoptive families in Georgia through post-adoptive services provided mothers with hope and a sense of support, reducing feelings of anxiety. Our camp study suggested that just coming to camp reduced anxiety. An important finding was the lack of correlation between the level of caregiver anxiety and severity of RAD symptoms. This was contrary to our hypothesis that severity of behavior would be associated with higher parent anxiety. Parent anxiety tended to be high regardless of behavior severity. Recognizing how elevated parent anxiety can be over, any behavioral concerns can be an important focus for therapists' empathy, psycho-education, and treatment. Even low level behavioral problems may seem very serious to parents who project poor life outcomes for their child and experience frustration in the parentchild relationship. Statistically significant reduction in parent stress was demonstrated on the state anxiety index, suggesting an increased sense of caregiver confidence for parenting for their children with RAD children.

\subsection{Immediate Impact of Camp}

The overall pre-post data clearly indicates that camp has a consistent and immediate effect on reducing perceived overt RAD associated behaviors. Consistency between the camp's locations suggests a reliable effect across settings and staff. Such consistency suggests a robust treatment program effect. There was a statistically significant effect on total RADQ scores across all the camps' locations with a slight variation in the calculated effect size. The variation in effect size could be due to the make-up of camp and the experience of camp's supporting staff. MI had the smallest effect size with the largest percentage of children having very severe RAD. CO and OR had the largest effect size which could be due to the fact that both camps were run multiple times and had veteran support staff.

When the effect was evaluated by severity level, it was the children in the severe group who showed the greatest immediate change while the children in the mild range had the smallest measured immediate effect. If this was merely due to "regression to the mean", then the very severe group should have shown the greatest improvement, but this was 
not the case. The result suggested that while the camp helped across severity levels, it was particularly helpful for children in the severe range. One hypothesis for this is that the behavior change may be more obvious than for moderate and mild children, and easier than for severe children who may have mental issues that are more intractable. This is a finding that requires confirmation and further study.

Effect size was also calculated for the critical items Table (7). There was a significant effect on the critical items based on severity rank as measured using Cohen's $d$. The most significant effect on the critical items was observed in the very severe rank. This is slightly different from the trend observed when all items of the RADQ were included. This suggests that programming at camp did an excellent job at modifying these specific behaviors.

\subsection{Long Term Impact of Camp}

Longer term follow up data indicated that improvements in behavior remained positive over a period of six months to two years. Youth in the Moderate, Severe and Very Severe categories, all maintained their gains at long term follow up (compare scores in Tables (7 and 10) above). This suggested that caregivers maintained positive changes in their parenting approach and children experienced an enduring shift in their sense of safety and emotional connection with the family. Since long term follow up participation was at 50 percent, the results must be interpreted with some caution. Additional research is required to confirm these findings. The study by Makela and Vierikko [8] on Finnish Theraplay intensive treatment in 4-day camp type format showed additional improvements at both six month and two year intervals when the entire sample was included at each follow up. Our current study relied on voluntary caregiver response to electronic questionnaires for follow-up with caregivers spread out across North America, and thus, there was less control over the follow-up participation than for the Finnish study. The scores within the Mild category could not be reliably interpreted due to having only four subjects.

Examination of post-camp scores and follow-up scores suggested that improvements were maintained in child behavior. While behavior problems remained, scores indicated behavior significantly below the baseline levels at pretesting. Effect sizes were large at both immediate post-testing and at long term follow-up. Sample size in the Mild category was too small for a reliable comparison or effect size. Results failed to demonstrate the additional, progressive improvement shown by the Finland group [8]. This may be due to key differences. The theraplay intensives provided for high levels of community support and therapeutic follow-up and attachment camp families returned to variable levels of community and treatment support. Indeed, the success of "networking for post camp support" was rated to be the lowest among the camp components by the follow-up study participants. This suggested the need for revisions of the method of providing support after camp. It was used for revisions of the aftercare process and should be the subject of future research.

Camp components of parent training, learning by watching, hands-on practice with staff support, trust building and gaining ideas for parenting were all rated as highly effective and helpful. Partner bonding and team work were predominantly in a positive direction (68\% reported sustained improvement), but this still received lower ratings than other components of the camp. A small percentage (14\%) of partners reported regression to the status quo in their relationship.

This is the only quantitative study we know of on North American camps focused specifically on attachment therapy programming for entire families. Purvis et al. [9] conducted attachment and sensory rich day camps for at risk adoptive children, demonstrating positive results even though parents and siblings had limited involvement. Results of the week-long, intensive, whole family camps under review for this current study were very positive and consistent with a similar concept applied in Finland. Results demonstrated the therapeutic value of this intensive format, and results also suggested ways to improve the effectiveness of this type of program. First, post-camp networking support should be improved for maximizing gains from camp. This could include increasing connection of families to support groups, increasing access or connection to therapists trained in attachment based therapy techniques and in trauma therapy, and increased access to professional parenting consultation. Second, attention should be given to sustaining caregiver team work and emotional connection. Children with intense emotional-behavioral needs place stress on partner relationships, and thus, sustaining improvements from camp may require connecting caregiver partners to local couple therapy supports or consultation.

Since this study was completed, the camp organizers have made changes to reflect the above suggestions, including a seven-week interactive internet support program. This is conducted as group parent consultation. A video module for reinforcing the lessons learned at camp is also provided. Caregivers have provided very positive qualitative feedback on 
this change, indicating that it has helped with maintaining their parenting changes and preventing a sense of isolation. Future research is recommended to examine parents' response to long-term camp's. The follow up program has the potential for providing various benefits to the parents that could positively impact family functioning. For instance, support can serve to diminish anxiety, boost confidence and enhance resiliency. It may increase adherence to the treatment model, thus increasing follow through on parent-child relationship building activities.

\section{CONCLUSION}

Findings have implications for treatment and research across disciplines. Attachment focused treatment activities were integrated with a culturally popular form of education and recreation, namely summer camp. An area for future qualitative research is whether removing treatment from a strict medical model made it more acceptable for participation by the entire family. A variety of pedagogical strategies were blended from the perspective of educational and behavioral psychology, including modeling, coaching, group discussion, small group problem solving and formal lecture. This was necessary to appeal to a diversity of learning styles, developmental levels and emotional needs. Qualitative research may be helpful in examining the relative effectiveness of activities and approaches in fostering relationship change, retention of learned skills and program satisfaction. Social work and juvenile justice workers need effective programs that restrain costs, promote social welfare, and reduce risk for multiple family members. This study demonstrated that a camp based model that includes the whole family and is of short term can have lasting positive effects. Additional research is needed to evaluate the degree to which the program promotes resiliency in family members, including reduced mental health risk for siblings. Furthermore, there was qualitative evidence from parent reports that the intensive change and education gave some parents confidence to become support for other parents or organizers of better services within their community. Qualitative research on this type of positive downstream effect could elucidate the possible community mental health benefits.

\section{ETHICS APPROVAL AND CONSENT TO PARTICIPATE}

Not applicable.

\section{HUMAN AND ANIMAL RIGHTS}

No Animals/Humans were used for studies that are base of this research.

\section{CONSENT FOR PUBLICATION}

Not applicable.

\section{CONFLICT OF INTEREST}

The authors declare no conflict of interest, financial or otherwise.

\section{ACKNOWLEDGEMENTS}

Declared none.

\section{REFERENCES}

[1] Zilberstein K. Clarifying core characteristics of attachment disorders: A review of current research and theory. Am J Orthopsychiatry 2006; 76(1): 55-64.

[http://dx.doi.org/10.1037/0002-9432.76.1.55] [PMID: 16569127]

[2] Chugani HT, Behen ME, Muzik O, Juhász C, Nagy F, Chugani DC. Local brain functional activity following early deprivation: A study of postinstitutionalized Romanian orphans. Neuroimage 2001; 14(6): 1290-301. [http://dx.doi.org/10.1006/nimg.2001.0917] [PMID: 11707085]

[3] Greenberg MT. Attachment and psychopathology in childhood. In: Cassidy J, Shaver PR, Eds. Handbook of attachment: Theory, research, and Clinical Applications. New York: Guilford Press 1999; pp. 469-96.

[4] Lieberman AF, Zeanah CH. Contributions of attachment theory to infant-parent psychotherapy and other interventions with infants and young children. In: Cassidy J, Shaver PR, Eds. Handbook of Attachment: Theory, Research, and Clinical Applications. New York: Guilford Press 1999; pp. 555-74.

[5] Booth PB, Jernberg AM. Theraplay: Helping parents and children build better relationships through attachment-based play. John Wiley \& Sons, Inc: San Francisco, CA 2010.

[6] Nielsen LE. Trust-based relational intervention (TBRI) for adopted children receiving therapy in an outpatient setting. Psychology Honors 
Projects. [Available from: http://digitalcommons.iwu.edu/psych_honproj/165/2014. Digital Commons @ IWU

[7] Cohen JA, Berliner L, Mannarino A. Trauma focused CBT for children with co-occurring trauma and behavior problems. Child Abuse Negl 2010; 34(4): 215-24.

[http://dx.doi.org/10.1016/j.chiabu.2009.12.003] [PMID: 20304489]

[8] Makela J, Vierikko I. From heart to heart: Interactive therapy for children in care report on the theraplay project in SOS children's villages in Finland 2001-2004. Espoo, Finland: SOS Children's Village Association 2004.

[9] Purvis KB, Cross DR, Federici R, Johnson D, McKenzie LB. The hope connection: A therapeutic summer day camp for adopted and at-risk children with special socio-emotional needs. Adopt Foster 2007; 31(4): 39-48. [http://dx.doi.org/10.1177/030857590703100406]

[10] Families by Design. Camps Colorado: Nancy Thomas Parenting; [Available from: http:/www.attachment.org/ caregivers/camps/2015.

[11] Randolph EM. Manual for the randolph attachment disorder questionnaire. $3^{\text {rd }}$ ed. Evergreen, CO: The Attachment Center Press 2000.

[12] Spielberger CD, Gorsuch RL, Lushene R, Vagg PR, Jacobs GA. Manual for the state-trait anxiety inventory. Palo Alto, CA: Consulting Psychologists Press 1983.

[13] Lyons-Ruth K, Jacobvitz D. Attachment disorganization: Unresolved lost, relational violence, and lapses in behavioral and attentional strategies. In: Cassidy J, Shaver PR, Eds. Handbook of Attachment: Theory, Research, and Clinical Applications. New York: Guilford Press 1999; pp. 520-54.

[14] Berlin LJ, Cassidy J. Relations among relationships: Contributions from attachment theory and research. In: Cassidy J, Shaver PR, Eds. Handbook of Attachment: Theory, Research, and Clinical Applications. New York: Guilford Press 1999; pp. 688-712.

[15] Goldman D. Social intelligence: The new science of human relationships. New York: Bantam Books 2006.

[16] Wimmer J, Vonk M, Reeves P. Adoptive mothers' perceptions of Reactive Attachment Disorder therapy and its impact on family functioning. Clin Soc Work J 2010; 38(1): 120-31. [http://dx.doi.org/10.1007/s10615-009-0245-x]

\section{(C) 2017 Coleman and Coleman.}

This is an open access article distributed under the terms of the Creative Commons Attribution 4.0 International Public License (CC-BY 4.0), a copy of which is available at: https://creativecommons.org/licenses/by/4.0/legalcode. This license permits unrestricted use, distribution, and reproduction in any medium, provided the original author and source are credited. 